\title{
Automatic Vehicle Over speed Controlling System using Microcontroller Unit and ARCAD
}

\author{
Prof. Madhuri Zambre \\ Professor, Department of Electrical Engineering, Modern College of Engineering Pune India \\ madhurizambre01@gmail.com
}

\begin{tabular}{|l|l|}
\hline Article History & \multicolumn{1}{|c|}{ Abstract } \\
$\begin{array}{l}\text { Article Submission } \\
18 \text { August } 2016 \\
\text { Revised Submission } \\
30 \text { October 2016 } \\
\text { Article Accepted } \\
22 \text { November 2016 } \\
\text { Article Published } \\
31^{\text {st }} \text { December 2016 }\end{array}$ & $\begin{array}{l}\text { The main purpose of this paper is to develop a system that avoid accidents because of } \\
\text { vehicles with high speed. Also, authorizes the pedestrians and public to cross the } \\
\text { highway road with no risk from vehicles which are moving with high speed. } \\
\text { Generally, drivers of the vehicles with high speed drives in an uncontrollable manner } \\
\text { even in speed limited areas without taking into consideration about the public. Even } \\
\text { traffic policemen could not able to control them and attain good response from the } \\
\text { high speed vehicle drivers. Also, we cannot monitor them to limit their speed at all } \\
\text { times in those areas. So, here we develop a system which controls the speed of the } \\
\text { vehicles in those locations without involving the drivers. Here, we employ RF } \\
\text { communication method in controlling the speed of the vehicles. Finally, we connect } \\
\text { RF receiver to the vehicle and locations are connected with the transmitter. Also, the } \\
\text { transmitters are coded to send the transmitted signals continuously with a small } \\
\text { delay between the adjacent signals. So, if a high speed vehicle is entering the location } \\
\text { then their receivers will automatically senses the received code. Thereafter, the speed } \\
\text { of the vehicle is limited dynamically by employing an embedded system inside them. } \\
\text { Keywords: RF, Transmitter, Receiver, Solar panel, Microcontroller, Gear motor, } \\
\text { relay, Proteus Software, ARCAD Software. }\end{array}$ \\
\end{tabular}

\section{Introduction}

With the advancement of the technology, high speeds vehicles arrived in the market which resulted in the accident and traffic rule violation. It is necessary to track the speed of vehicles. Tracking of these high speed vehicles became a concern for a traffic control authority. In infrared vehicle tracking system, problem occurs when two vehicles run simultaneously; this problem can be eliminated by using RFID tracking system. Global positioning system is a costlier system to implement and its maintenance is also high and it will not work well while vehicle is running under the tunnel. This problem is also eliminated by RFID. RFID device provides tags that help in the vehicle tracking. Employing RFID tag in a vehicle line certifies functionality is optimum, efficiency is improved, also avoids the chance of theft and fraud.

Radio frequency is the one which gives the rate of oscillation around the range from $3 \mathrm{kHz}$ to $300 \mathrm{GHz}$ that is related to radio wave frequencies. RF always deals with electrical instead of mechanical oscillations. Moreover, RF system which is mechanical also exists. Also, the term radio frequency is abbreviated as RF which is similar to radio. It is used to express communication in wireless medium which is complementary to communication through cables. We also know that to receive these radio waves an antenna system is necessary. Moreover, a receiving antenna receives many radio waves at an instance, but a tuner helps in tuning to a specific frequency range. This job is accomplished by a resonator which is also called as a tuned circuit having a capacitor, inductor in its simplest form. It increments oscillations within the spectrum and limits oscillations outside this spectrum. 
Radio communications distance specifically depends on rather than wavelength like power transmitted, quality of the received signal, size, antenna height, interference. Radio propagation is the one where the radio waves are propagated to the receiver after transmitted from the transmitter. Also, Ground waves, sky waves transmit the signal to very long distances in comparison with that of space wave propagation. It also helps in approximating the distance between the Tx and $\mathrm{Rx}$.

\section{Related Work}

The embedded researchers are developing a system that decrements the violations in the traffic rules. Driving violation and behavior of the driver is known, thereby conscious and secured driving is attained. If the violations are repeated continuously, then it results in much high penalty that causes in the decrease of crossing the speed limit by a driver in the vehicle. So, it is attained by employing zig-bee that come up with transmission of information which is cost effective. GSM modern transmits the information to the radio station immediately after violation of rules by the vehicle. We can place traffic signs to follow road rules. RFID tags are attached to the vehicles for initiating licensed access to parking in a specific slot. It also activates CC cameras if an automobile leaves or enters the location which is under control. Every initiation is recorded in a server's database to know the past activities that are accessed and billing is done on daily basis. It also gives the notification immediately if an automobile exits or enters the parking lot. It totally avoids manual recording of keeping which increases exactness and productivity of the staff. The conventional approach is shown in figure 1.
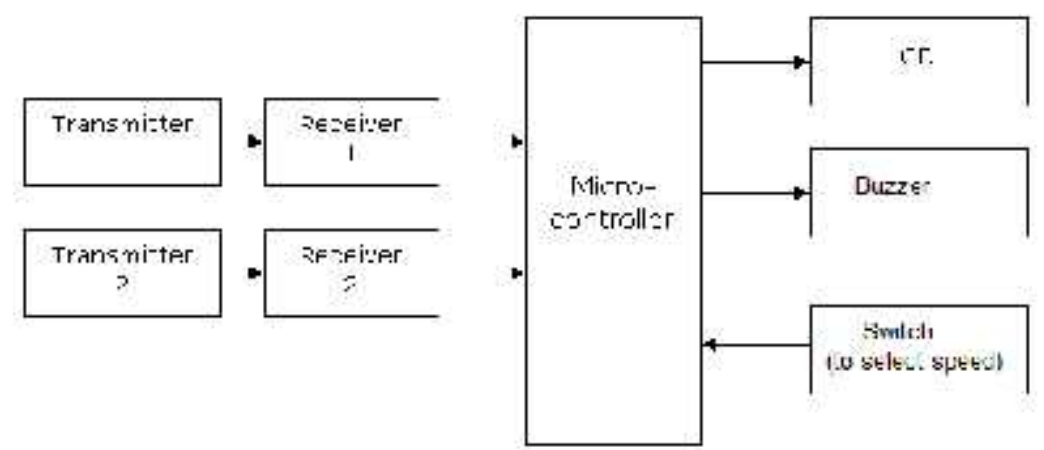

Fig 1: Existing vehicle over speed controlling system.

\section{Proposed Automatic Vehicle Overspeed Controlling System}

The block diagram of the proposed concept is shown in below figures. In both transmitter and receiver, the microcontroller used is I 6F877A. It is a 40 pin IC and it uses flash memory to write and erase the data. The regulator power connected to an embedded system. When the vehicle speed exceeds, a message will be observed on the screen of the LCD. It consists of two registers such as data, command. The relay is connected with the Microcontroller and when the relay switch is ON, lamp will glow. The brightness of the automatically increase or decrease according to the daylight. The opto-coupler is a passive device which is mainly employed in branching. It is a mixture of source of light and a detector which is photosensitive. 


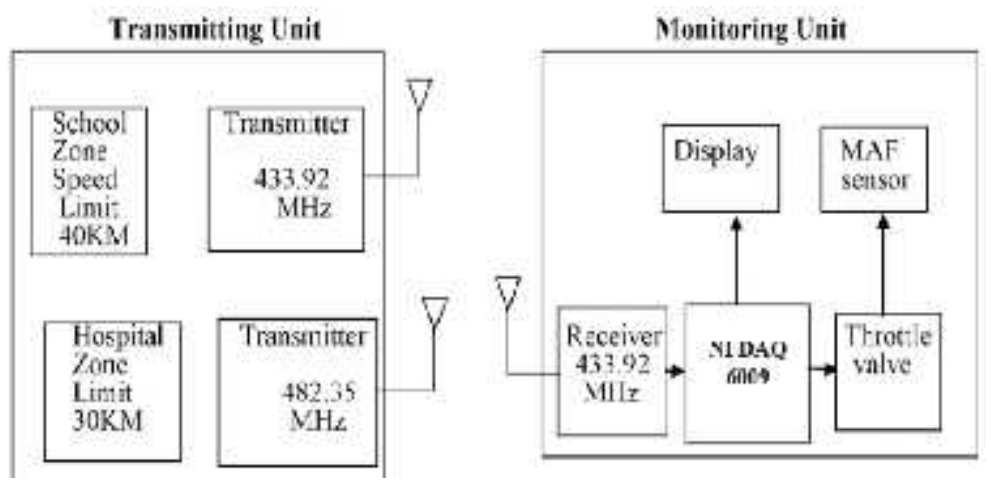

Fig 2: Proposed vehicle over speed controlling system.

At the receiver part, the microcontroller 16F877A is employed. The gear motor is used at the receiver side which is attached along with the vehicle. $10 \mathrm{rpm}, 12 \mathrm{~V} \mathrm{DC}$ gear motor is employed. When the motor rotates, the solar panel tilt at an angle of 180 degree. The OEM solar panels are used. The charge of the solar panel is given to the battery through the charging circuit. Here, $12 \mathrm{~V}$ lithium batteries is used. When the vehicle entering into certain zones such as school zones, hospital zones, hair pin bend, steep zones, accident prone areas etc and when the speed of the vehicle is exceeding the limited speed, warning message will be displayed in the LCD and speed of the vehicle will be automatically reduced without the interruption of the driver. The flowchart is given in figure 3.

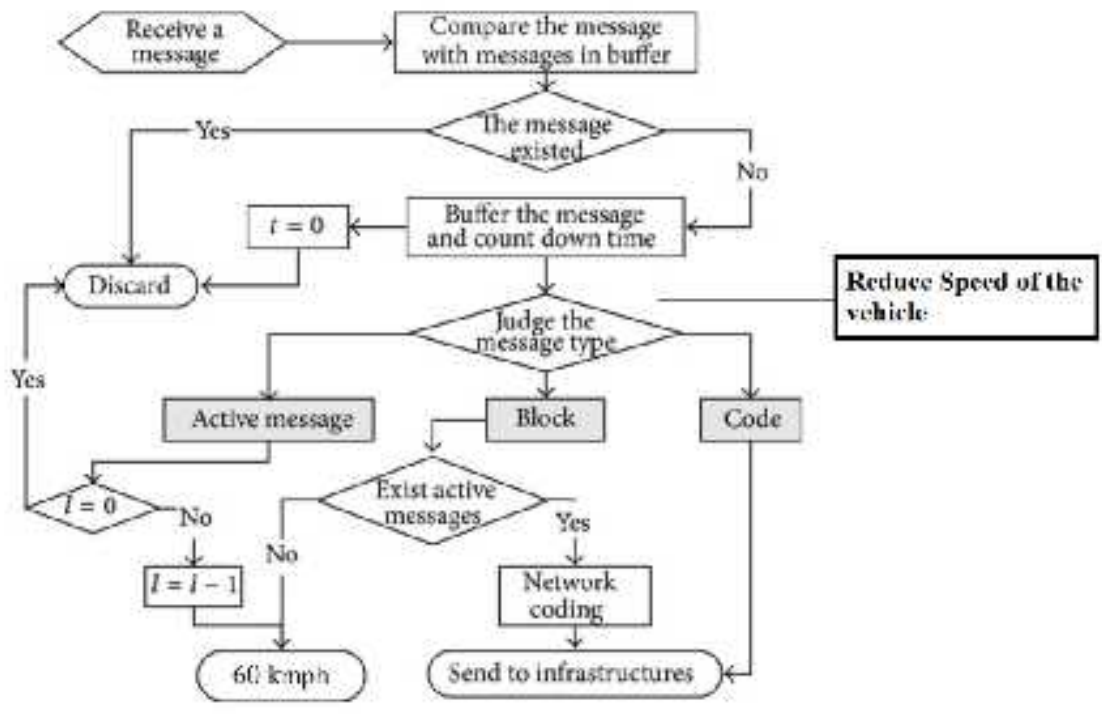

Fig 3: Flowchart of vehicle over speed controlling system.

PIC16F877A microcontroller is becoming more and more popular in recent a day which is employed by startups and professional companies. It is very simple that employs the technique of flash memory because of write-erase many hundred times. It is superior than RISC Microcontroller in terms of speed and programming the code. It has many applications and they are embedded in automobile industries, home automation systems, sensing of remote areas, security systems etc. It is applicable for smart tags and also for devices which employs a battery system due to low consumption of power. EEPROM is a memory element that is applied to systems in which everlasting storage of components are necessary. It is more flexible and easy to install. This ability of PIC $16 \mathrm{~F} 877 \mathrm{~A}$ is employed to develop assembly-line or to maximize operations on end products. 


\section{Hardware and Simulation Results}

ARCAD Software is used for designing purpose. PROTEUS SOFTWARE combines advanced schematic capture, mixed mode SPICE simulation, PCB layout and auto routing to make a complete electronic design system. The Schematic Capture an easy to use yet and extremely powerful tool for entering the design. PROSPICE Mixed mode SPICE Simulation industry standard SPICE3F5 simulator upgradeable to unique virtual system modeling technology and ARES PCB Layout.

An OEM Solar Module kit in a site consists of overall site, solar panel. SWIL6 is a regulator in terms of voltage having a cable at the output. Solar panel can tilt by an angle 180 degree. Solar panels with high efficiency are developed to move out from the standards of an industry that makes it reliable and produces high output power. Features are it provides exceptional reliability and maximum power output and also $12 \mathrm{~V}$ I8Ah battery included with kit.

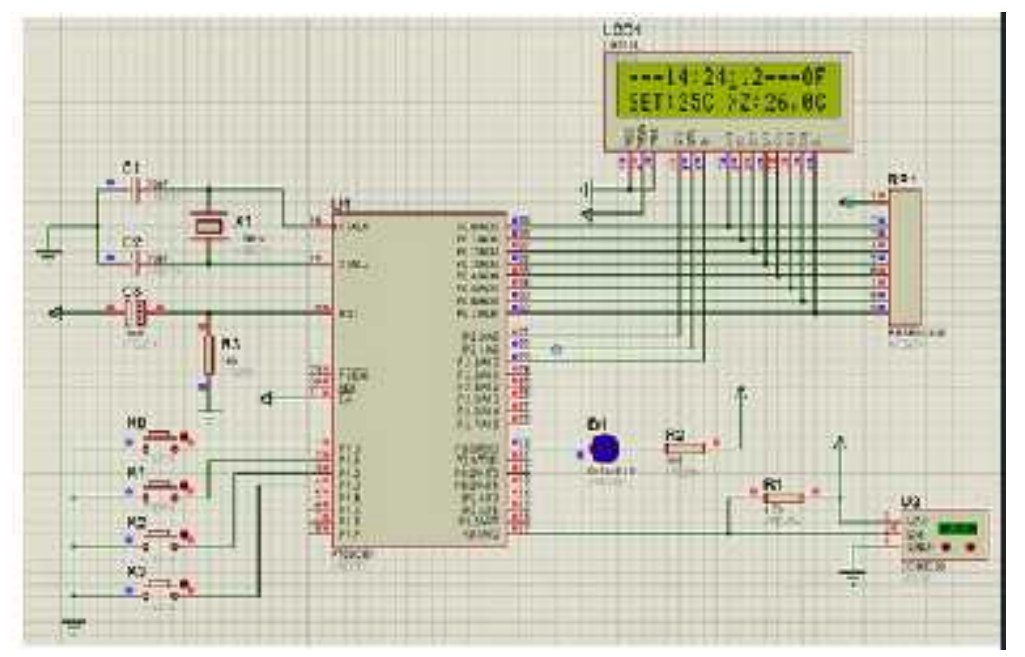

Fig 4: Circuit schematic of vehicle over speed controlling system.

The 10rpm 12v DC gear motor is employed to produce great force although in terms of low speed. Decreases the speed of the motor and complexity of design and RPM. Relays are majorly employed in automobiles. They are available in varied sizes and different rating. Also, they are employed in remote control switches. Any specific vehicle will have at least 20 relays. These are again commanded by an other switch. Relays permit low current to hold a circuit where high current is flowing. It is the one which is an electronic display module and employed in many applications. A $16 \times 2 \mathrm{LCD}$ is the standard one which is employed in different circuits. They are superior to seven segments display. They are very simple to install and easily coded.

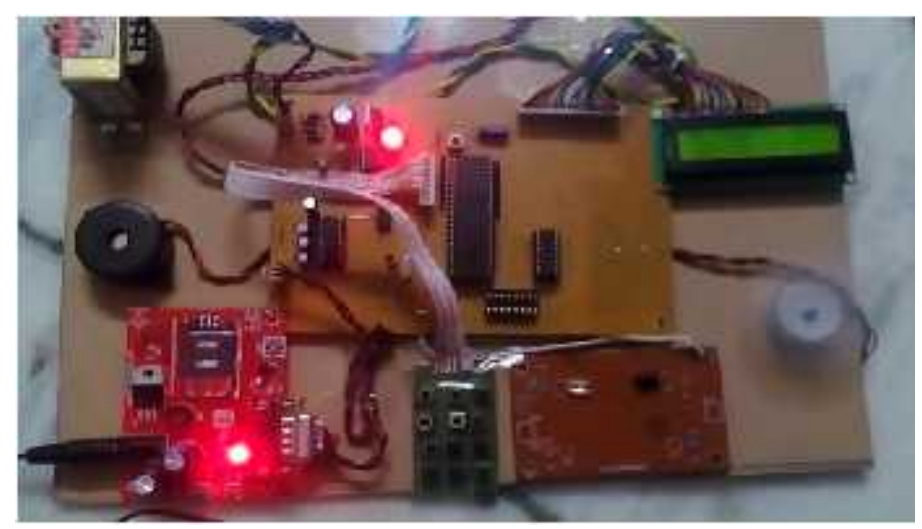

Fig 5: Hardware setup of vehicle over speed controlling system. 


\section{Conclusion}

This paper explains about the automatic over speed control of the vehicle by using the RF. The RF transmitter is placed over certain zones such as school zones, hospital zones etc and RF receiver is fixed along with the vehicle so that the speed of the vehicle can be controlled automatically without the interruption of the driver. Also the brightness of the lamp can be automatically increase or decrease automatically according to the daylight. Thus this proposed system in real time can avoid the accidents due to high speed vehicles.

\section{References}

[1] H. Sun, Z. Hou and D. Li, "Coordinated Iterative Learning Control Schemes for Train Trajectory Tracking With Overspeed Protection," in IEEE Transactions on Automation Science and Engineering, vol. 10, no. 2, pp. 323-333, April 2013, doi: 10.1109/TASE.2012.2216261.

[2] A. Doshi, S. Y. Cheng and M. M. Trivedi, "A Novel Active Heads-Up Display for Driver Assistance," in IEEE Transactions on Systems, Man, and Cybernetics, Part B (Cybernetics), vol. 39, no. 1, pp. 8593, Feb. 2009, doi: 10.1109/TSMCB.2008.923527.

[3] C. Shi, C. Guo and C. Sun, "Simulation and Optimal Control of Diesel Engine Propulsion System," 2009 WRI World Congress on Computer Science and Information Engineering, Los Angeles, CA, 2009, pp. 87-91, doi: 10.1109/CSIE.2009.46.

[4] J. Dai, S. W. Nam, M. Pande and G. Esmaeili, "Medium-Voltage Current-Source Converter Drives for Marine Propulsion System Using a Dual-Winding Synchronous Machine," in IEEE Transactions on Industry Applications, vol. 50, no. 6, pp. 3971-3976, Nov.-Dec. 2014, doi: 10.1109/TIA.2014.2316361.

[5] Ma Zengqiang, Pan Cunzhi and Wang Yongqiang, "Road Safety Evaluation from Traffic Information Based on ANFIS," 2008 27th Chinese Control Conference, Kunming, 2008, pp. 554-558, doi: 10.1109/CHICC.2008.4605560.

[6] T. D. Barkand, "Application of a suspension rope brake to a single rope mine hoisting system," Conference Record of the 1992 IEEE Industry Applications Society Annual Meeting, Houston, TX, USA, 1992, pp. 2041-2046 vol.2, doi: 10.1109/IAS.1992.244199.

[7] W. Hongjian, T. Yuelin and L. Zhi, "RFID Technology Applied in Highway Traffic Management," 2010 International Conference on Optoelectronics and Image Processing, Haikou, 2010, pp. 348-351, doi: 10.1109/ICOIP.2010.110.

[8] Ma Zengqiang, Gao Guosheng, Song Wanmin and Yan Yan, "Wireless monitoring system of vehicle overspeed on freeway based on GPRS," 2008 27th Chinese Control Conference, Kunming, 2008, pp. 550-553, doi: 10.1109/CHICC.2008.4605559.

[9] Ying-ji Liu, Xu Liu and Yu Yao, "Overspeed events multiple sections research based on clustering algorithm," Proceedings 2011 International Conference on Transportation, Mechanical, and Electrical Engineering (TMEE), Changchun, 2011, pp. 1564-1567, doi: 10.1109/TMEE.2011.6199507.

[10] J. Ward, S. Worrall, G. Agamennoni and E. Nebot, "Vehicle operation safety monitoring using context based metrics: A case study," 2013 IEEE Intelligent Vehicles Symposium (IV), Gold Coast, QLD, 2013, pp. 19-24, doi: 10.1109/IVS.2013.6629441. 